\title{
Coherent and Incoherent Quantum Stochastic Resonance
}

\author{
Milena Grifoni and Peter Hänggi \\ Institut für Physik, Universität Augsburg, Memminger Strasse 6, D-86135 Augsburg, Germany
}

(Received 26 July 1995)

\begin{abstract}
We study quantum stochastic resonance (QSR) in the periodically driven biased spin-boson system. The amplitude and the phase of the nonlinear response function vs temperature are studied in the incoherent and coherent tunneling regimes, encompassing adiabatic and nonadiabatic driving, weak and strong Ohmic coupling $\alpha$. For $\alpha=1 / 2$ we present exact QSR results. As a general feature, a principal maximum appears when the static asymmetry exceeds the driving frequency and strength. Moreover, the nonlinear QSR shows a noise-induced suppression of higher harmonics and a characteristic phase shift.
\end{abstract}

PACS numbers: 05.45.+b, 03.65.Sq

The process whereby noise operates on a bistable system enhancing the response to an external periodic signal has been termed stochastic resonance (SR) [1]. Since its discovery in 1981, this intriguing phenomenon has been the object of many investigations [2,3]. Classically, the resonance condition is assumed when the thermal hopping frequency is near the frequency of the modulation. Upon decreasing the temperature, quantum tunneling becomes increasingly important. Its role has only started to be explored. Above a crossover temperature $T_{0}$, thermally activated transitions dominate over quantum tunneling events [4]. The effects of quantum noise then result in a quantum correction factor of the classical rate of activation [5]. As the temperature is decreased below $T_{0}$, tunneling transitions prevail. The aim of our study herein is the exploration of quantum stochastic resonances in the deep quantum regime $0 \leq T \ll T_{0}$. The quantum noise is characterized by the temperature of the thermal bath and by the coupling strength of the bistable system to the environment. In the absence of driving, at sufficiently high temperatures and/or coupling, the dissipative effects are so strong that quantum coherence is completely suppressed by incoherent tunneling transitions. In this case the dynamics can be modeled by rate equations. This picture still holds for low-frequency driving [6].

The role of incoherent transitions at low-frequency driving for classical SR has been addressed first in Ref. [7] and its extension to quantum stochastic resonance (QSR) in [8]. In [8] it is found that QSR succeedingly vanishes as the equality between forward and backward transitions is approached. In contrast, for classical SR, the resonance is maximal in the $a b$ sence of asymmetry [9]. As the external frequency is increased and/or when the temperature is lowered, quantum coherence and/or driving-induced correlations render the dynamics intrinsically non-Markovian [10]. In this work we predict various novel QSR phenomena, both for coherent (conventional quantum coherent regime for adiabatic driving and low temperatures; driving-induced coherent regime for nonadiabatic driving) and incoherent driven quantum dynamics. Apart from the generic amplification of the first harmonic of the periodic quantum output, we also discover the existence of the quantum analog of noise-induced suppressions of higher harmonics [9], and, correspondingly, a discontinuity in the phase shift between periodic response and applied driving signal.

As a working model we consider a two-level-system (TLS) Hamiltonian. Here the bath is described as an ensemble of harmonic oscillators with a bilinear coupling in the TLS-bath coordinates, and we allow for an externally applied harmonic force $(\hbar \hat{\boldsymbol{\epsilon}} / a) \cos \Omega t$

$$
\begin{aligned}
H= & -\frac{\hbar}{2}\left(\Delta \sigma_{x}+\epsilon_{0} \sigma_{z}\right)-\frac{\hbar \hat{\epsilon}}{2} \cos (\Omega t) \sigma_{z} \\
& +\frac{1}{2} \sum_{\alpha}\left(\frac{p_{\alpha}^{2}}{m_{\alpha}}+m_{\alpha} \omega_{\alpha}^{2} x_{\alpha}^{2}-c_{\alpha} x_{\alpha} a \sigma_{z}\right) .
\end{aligned}
$$

The $\sigma$ 's are Pauli matrices, and the eigenstates of $\sigma_{z}$ are the basis states in a localized representation, while $a$ is the tunneling distance. The tunneling splitting energy of the symmetric TLS is given by $\hbar \Delta$, while the asymmetry energy is $\hbar \epsilon_{0}$. As far as the properties of the TLS are concerned, the coupling to the bath is fully characterized by the correlation functions of the stochastic force $\xi(t)=$ $\sum_{\alpha} c_{\alpha} x_{\alpha}$ in thermal equilibrium [11,12],

$$
\langle\xi(t) \xi(0)\rangle_{\beta}=\frac{\hbar}{\pi} \int_{0}^{\infty} d \omega J(\omega) \frac{\cosh [\omega(\hbar \beta / 2-i t)]}{\sinh (\omega \hbar / 2)},
$$

and $\langle\xi(t)\rangle_{\beta}=0$, where $\beta=1 / k_{B} T$ is the inverse of the temperature. $J(\omega)$ is the spectral density of the environment, and we make the specific choice of Ohmic dissipation $J(\omega)=\left(2 \pi \hbar / a^{2}\right) \alpha \omega e^{-\omega / \omega_{c}}$, where $\alpha$ is the dimensionless coupling strength and $\omega_{c}$ is a cutoff frequency [11,12]. The dynamical quantity of interest is the quantum expectation value $P(t)=\left\langle\sigma_{z}(t)\right\rangle$. The combined influence of dissipative and driving forces at intermediate times results in a reduction of the coherent tunneling motion of the isolated TLS by incoherent tunneling transitions. For times $t$ large compared to the time scale of the transient dynamics, the motion acquires the periodicity of the external perturbation, and $P(t)$ reaches the 
asymptotic value

$$
\lim _{t \rightarrow \infty} P(t)=P^{(\mathrm{as})}(t)=\sum_{m=-\infty}^{\infty} P_{m}(\Omega, \hat{\epsilon}) e^{-i m \Omega t} .
$$

The harmonics $P_{m}$ of $P(t)$ present the quantity of interest to investigate the nonlinear QSR. In the linear response approximation, only the harmonics $0, \pm 1$ of $P^{(\text {as) }}(t)$ are different from zero, $P_{0}$ being just the thermal equilibrium value in the absence of driving and $P_{ \pm 1}=\hbar \hat{\epsilon} \chi( \pm \Omega)$ being related to the linear susceptibility $\chi(\Omega)$ by Kubo's formula. With increasing strength $\hat{\boldsymbol{\epsilon}}$ higher harmonics become important. The amplitudes $\left|P_{m}\right|$ determine the weights of the $\delta$ spikes of the averaged spectral power density in the asymptotic state $S^{(\text {as) }}(\omega)$ via the relation [13]

$$
S^{(\text {as })}(\omega)=2 \pi \sum_{m=-\infty}^{\infty}\left|P_{m}(\Omega, \hat{\epsilon})\right|^{2} \delta(\omega-m \Omega) .
$$

Thus, in order to investigate QSR we shall examine the power amplitude $\eta_{m}$ in the $m$ th frequency component of
$S^{(\mathrm{as})}(\omega)$ and the associated phase shift $\varphi_{m}$ as well, i.e.,

$$
\begin{aligned}
\eta_{m}(\Omega, \hat{\boldsymbol{\epsilon}}) & =4 \pi\left|P_{m}(\Omega, \hat{\boldsymbol{\epsilon}})\right|^{2}, \\
\varphi_{m}(\Omega, \hat{\boldsymbol{\epsilon}}) & =\arctan \left[\frac{\operatorname{Im} P_{m}(\Omega, \hat{\boldsymbol{\epsilon}})}{\operatorname{Re} P_{m}(\Omega, \hat{\boldsymbol{\epsilon}})}\right] .
\end{aligned}
$$

In doing so we shall take advantage of novel results for the dynamics of driven-dissipative two-state systems $[6,10,14]$. An exact formal solution for any strength of the external force can be obtained using a real-time path integral approach. It is in the form of a power series in the tunneling transitions. The series can be summed up exactly in analytic form for the special value $\alpha=1 / 2$ of the Ohmic strength, to give

$$
P_{m}(\Omega, \hat{\epsilon})=\frac{\gamma}{\gamma-i m \Omega} \frac{2 \omega_{c}}{\pi} h_{m}(-i m \Omega, \gamma),
$$

with

$$
\begin{aligned}
h_{2 k}(\lambda, \gamma) & =(-1)^{k} \sin (\pi \alpha) \int_{0}^{\infty} d \tau e^{-\lambda \tau-\gamma \tau / 2-S(\tau)} e^{-i k \Omega \tau} \sin \left(\epsilon_{0} \tau\right) J_{2 k}\left(\frac{2 \hat{\epsilon}}{\Omega} \sin \frac{\omega \tau}{2}\right), \\
h_{2 k+1}(\lambda, \gamma) & =(-1)^{k} \sin \pi \alpha \int_{0}^{\infty} d \tau e^{-\lambda \tau-\gamma \tau / 2-S(\tau)} e^{-i(k+1 / 2) \Omega \tau} \cos \left(\epsilon_{0} \tau\right) J_{2 k+1}\left(\frac{2 \hat{\epsilon}}{\Omega} \sin \frac{\Omega \tau}{2}\right) .
\end{aligned}
$$

Here, $\left.S(\tau)=2 \alpha \ln \left(\hbar \beta \omega_{c} / \pi\right) \sinh (\pi \tau / \hbar \beta)\right], J_{m}(z)$ is a Bessel function of the first kind, and $\gamma=\pi \Delta^{2} / 2 \omega_{c}$ is the renormalized tunneling frequency $\Delta_{e}$ [see Eq. (11) below] for $\alpha=1 / 2$. In Fig. 1 the fundamental amplitude $\eta_{1}$ is plotted as a function of the temperature for different asymmetries $\epsilon_{0}$. It exhibits a bell-shaped maximum only when the static asymmetry exceeds both the external frequency and strength. A double peak appears at the lowest temperatures in the curve with $\hat{\boldsymbol{\epsilon}}=\Omega=\epsilon_{0} / 2$ (middle curve). Also, in the associated phase shift $\varphi_{1}$ the double resonance structure is observed (not shown). In the inset, $\eta_{1}(\Omega)$ is depicted. In contrast to the undriven case (at $\alpha=1 / 2$ ) where only incoherent tunneling occurs, we find a driving-induced coherence in the temperature region where the secondary peak of $\eta_{1}(T)$ appears, as shown by the presence of resonances near submultiples of $\epsilon_{0}$ (full line in the inset). As the temperature is increased, the coherence is lost (dashed line). In the temperature region where the SR maximum appears, incoherent transitions prevail.

In the static case $(\hat{\boldsymbol{\epsilon}}=0)$, the major difficulty for an analytic resummation of the series arises from the bathinduced correlations between different tunneling transitions. Further, it is known that a perturbative approach would fail in describing the destruction of the quantum coherent motion induced by the environmental stochastic forces at finite temperatures. In the presence of timedependent driving, the situation is even more intricate since one also has to take into account the correlations induced by the external field. The bath-induced correlations can be treated within the noninteracting-blip approximation (NIBA) for the stochastic force, whose range of validity has to be determined self-consistently [10]. A set of coupled equations for the Fourier coefficients $P_{m}$ can be derived for any strength and frequency of the driving force, yielding the non-Markovian result

$$
P_{0}=\frac{f_{0}(0)}{g_{0}(0)}-\sum_{m \neq 0} \frac{g_{-m}(0)}{g_{0}(0)} P_{m},
$$

and for $m \neq 0$

$$
P_{m}=\frac{i}{m \Omega}\left[f_{m}(-i m \Omega)-\sum_{m^{\prime}} g_{m-m^{\prime}}(-i m \Omega) P_{m^{\prime}}\right] \text {. }
$$

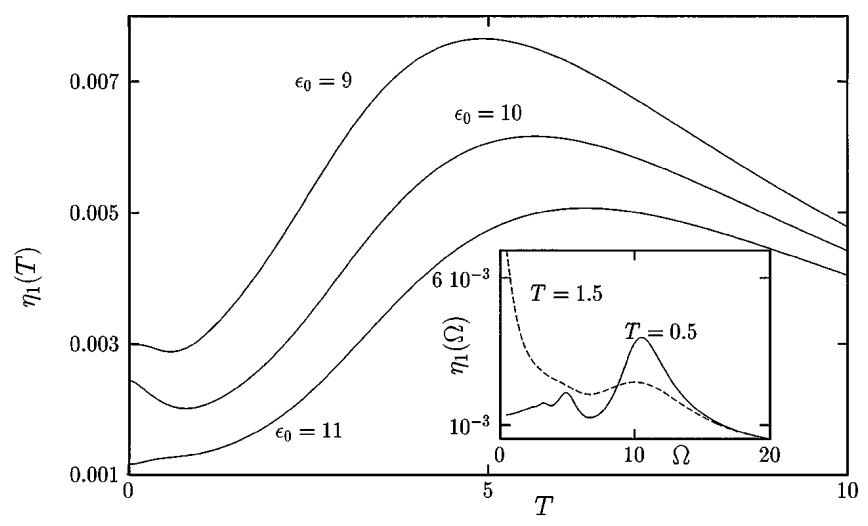

FIG. 1. Amplification vs temperature of the fundamental amplitude $\eta_{1}$ [cf. (5)], via quantum SR, for different asymmetries $\epsilon_{0}$ and $\hat{\epsilon}=\Omega=5$ in the exactly solvable case $\alpha=1 / 2$ of the Ohmic strength. The inset depicts $\eta_{1}$ vs driving frequency $\Omega$ at $\epsilon_{0}=10, \hat{\epsilon}=5$. The two temperatures show the transition from the coherent to the incoherent regime. Frequencies are given in units of $\gamma$, temperatures in units of $\hbar \gamma / k$. 
The coefficients $f_{m}$ are defined by Eq. (7) via $f_{m}=$ $\Delta^{2} h_{m}(-i m \Omega, 0)$; for the $g_{m}$ one has

$$
\begin{aligned}
g_{2 k}(\lambda)= & (-1)^{k} \Delta^{2} \cos \pi \alpha \int_{0}^{\infty} d \tau e^{-\lambda \tau-S(\tau)} \\
& \times e^{-i k \Omega \tau} \cos \left(\epsilon_{0} \tau\right) J_{2 k}\left(\frac{2 \hat{\epsilon}}{\omega} \sin \frac{\Omega \tau}{2}\right), \\
g_{2 k+1}(\lambda)= & (-1)^{k+1} \Delta^{2} \cos \pi \alpha \int_{0}^{\infty} d \tau e^{-\lambda \tau-S(\tau)} \\
& \times e^{-i(k+1 / 2) \Omega \tau} \sin \left(\epsilon_{0} \tau\right) J_{2 k+1}\left(\frac{2 \hat{\epsilon}}{\Omega} \sin \frac{\Omega \tau}{2}\right) .
\end{aligned}
$$

In the low-frequency regime $\Omega \ll \alpha k T / \hbar$, Eqs. (8) and (9) can be simplified because, to leading order, driving-induced non-Markovian correlations do not contribute. The asymptotic dynamics is intrinsically incoherent and governed by the equation $\dot{P}^{(\text {as })}(t)=$ $-\gamma_{\alpha}(t)\left[P^{(\text {as })}(t)-P_{\text {eq }}(t)\right]$, possessing the form of a rate equation with a time-dependent rate $\gamma_{\alpha}(t)=$ $\operatorname{Re} \Sigma[\varepsilon(t)]$ and time-dependent adiabatic equilibrium $P_{\text {eq }}(t)=-\tan (\pi \alpha) \operatorname{Im} \Sigma[\varepsilon(t)] / \operatorname{Re} \Sigma[\varepsilon(t)]$. Here, $\varepsilon(t)=\epsilon_{0}+\hat{\epsilon} \cos \Omega t$ plays the role of a time-dependent adiabatic asymmetry,

$\Sigma[\varepsilon(t)]\left(\frac{\hbar \beta \Delta_{e}}{2 \pi}\right)^{1-2 \alpha}=\frac{\Delta_{e}}{\pi} \frac{\Gamma(\alpha+i \hbar \beta \varepsilon(t) / 2 \pi)}{\Gamma(1-\alpha+i \hbar \beta \varepsilon(t) / 2 \pi)}$,

while $\Gamma$ denotes the gamma function and $\Delta_{e}=$ $\Delta\left(\Delta / \omega_{c}\right)^{\alpha /(1-\alpha)}[\cos (\pi \alpha) \Gamma(1-2 \alpha)]^{1 /(2-2 \alpha)}$. The rate equation can be solved in terms of quadratures to give

$$
\begin{aligned}
P_{m}(\Omega, \hat{\boldsymbol{\epsilon}})= & \frac{\Omega}{2 \pi} \int_{0}^{2 \pi / \Omega} d t_{0} \gamma_{\alpha}\left(t_{0}\right) P_{\mathrm{eq}}\left(t_{0}\right) e^{-i m \Omega t_{0}} \\
& \times \int_{0}^{\infty} d t_{1} e^{-i m \Omega t_{1}} \exp \left(-\int_{t_{0}}^{t_{0}+t_{1}} d t \gamma_{\alpha}(t)\right) .
\end{aligned}
$$

Equation (12) completely describes the nonlinear dynamics at low frequency within the validity range of NIBA. We observe that classical nonlinear SR has been recently investigated in a SQUID system [15]. Lowering further the temperature brings the system into the regime of Eq. (12) where nonlinear QSR could be observed for a macroscopic quantum system. At low frequencies, NIBA is justified for strong damping $\alpha>1$, while for weak coupling $\alpha<1$ it is valid in the region $\alpha k T \geq \hbar \Delta$ or $|\varepsilon(t)| \gg \Delta$. In the small coupling limit $\alpha \ll 1$ the relaxation rate in (12) becomes

$$
\gamma_{\alpha}(t)=\pi \alpha \Delta_{e}^{2} \frac{\varepsilon(t)}{(2 \pi \alpha k T / \hbar)^{2}+\varepsilon(t)^{2}} \operatorname{coth} \frac{\hbar \varepsilon(t)}{2 k T},
$$

and $P_{\text {eq }}(t)=\tanh [\hbar \varepsilon(t) / 2 k T]$. It should be noted that in the nonlinear regime $\hat{\epsilon} \gg \epsilon_{0}$ the presence of the term $2 \pi \alpha k T$ in the denominator is crucial since $\varepsilon(t)$ is zero at any instant $t$, obeying $t=1 / \Omega \operatorname{arcos}\left(\epsilon_{0} / \hat{\epsilon}\right)$.
This explicitly shows the breakdown of any perturbative approach to deal with time dependent problems at high temperatures in the nonlinear regime.

At low temperatures $\alpha k T \leq \hbar \Delta$ and weak coupling NIBA fails to predict the correct long-time behavior because the neglected bath-induced correlations contribute to the dissipative effects to first order in the coupling strength. Nevertheless, at low temperatures, where conventional quantum coherence occurs, a perturbative treatment is allowed [11,12]. A solution of the dynamics can then be discussed in the low-frequency regime $\hat{\epsilon} \Omega \ll \Delta^{2}$ where the tunneling motion may be treated in the adiabatic limit [14]. In the adiabatic regime and for weak coupling, $P^{(\text {as) }}(t)$ is simply related to the function $N(t)$, representing the population difference between the lower and upper eigenstates of the driven two-state system, by $P^{(\mathrm{as})}(t)=$ $\hbar \varepsilon(t) / E(t) N(t)$. Here $E(t)=\left[\Delta^{2}+\varepsilon(t)^{2}\right]^{1 / 2}$ is the timedependent level splitting. One then finds that $N(t)$ obeys the first order differential equation $\dot{N}(t)=-\gamma(t)[N(t)-$ $\left.N_{\text {eq }}(t)\right]$, which is again in the form of a rate equation with a time-dependent rate $\gamma(t)=\pi \alpha \Delta^{2} / E(t) \operatorname{coth} E(t) / 2 k T$ and time-dependent adiabatic equilibrium value $N_{\text {eq }}(t)=$ $\tanh E(t) / 2 k T$. Again this equation can be solved in terms of quadratures to give for the harmonics of $P^{(\text {as })}(t)$

$$
\begin{aligned}
P_{m}(\Omega, \hat{\boldsymbol{\epsilon}})= & \frac{\Omega}{2 \pi} \int_{0}^{2 \pi / \Omega} d t_{0} \frac{\hbar \varepsilon\left(t_{0}\right)}{E\left(t_{0}\right)} e^{-i m \Omega t_{0}} \\
& \times \int_{0}^{\infty} d t_{1} N_{\mathrm{eq}}\left(t_{1}\right) \gamma\left(t_{1}\right) \\
& \times \exp \left(-\int_{t_{0}}^{t_{0}+t_{1}} d t \gamma(t)\right) .
\end{aligned}
$$

Equations (12)-(14) capture the essential features of the dynamics of the driven TLS for low frequencies and weak coupling in the whole temperature range. For strong coupling, Eq. (12) holds down to the lowest temperatures. Figure 2 shows the behavior of $\eta_{3}$ vs temperature. We discover the quantum analog of noise-induced suppression

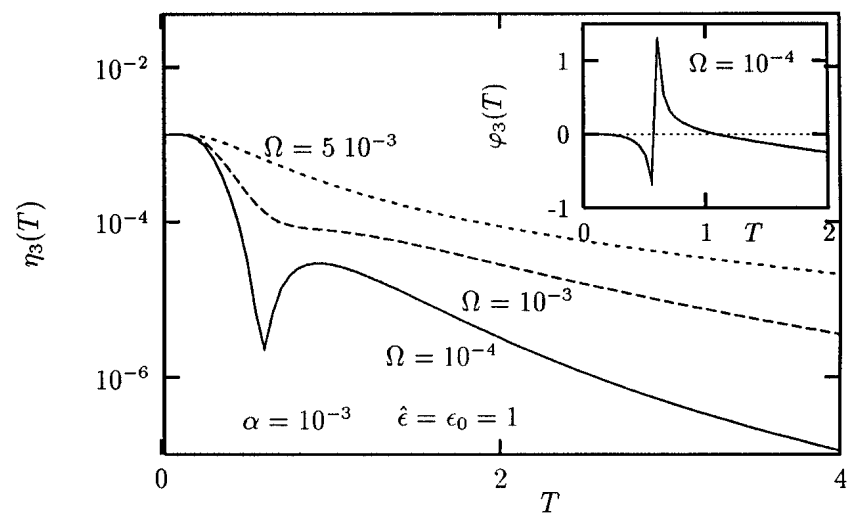

FIG. 2. Noise-induced suppression of the third amplitude $\eta_{3}$ vs temperature in the low-frequency regime (14) as the frequency is decreased. The inset depicts the discontinuity in the associated phase shift in correspondence to the NIS. Frequencies are given in units of $\Delta$, temperatures in units of $\hbar \Delta / k$. 
(NIS) of higher harmonics $[9,15]$ as the frequency is decreased. Correspondingly, in the inset the associated phase shift shows a sharp discontinuity at the very same value of the temperature where the NIS appears.

As the frequency is increased, non-Markovian correlations become increasingly important rendering the solution of Eqs. (8) and (9) extremely difficult. Nevertheless, a high-frequency approximation can be discussed when the condition $v\left(\epsilon_{0}\right) \ll \Omega$ is met [16]. Here, $v\left(\epsilon_{0}\right):=\lim _{\hat{\epsilon} \rightarrow 0} g_{0}(0)=\operatorname{Re} \Sigma\left(\epsilon_{0}\right)$ is the only nonvanishing coefficient $g_{m}$ in (10) for zero external field. It defines the time scale for the system to reach thermal equilibrium in the absence of driving. To leading order we then find

$$
\begin{aligned}
P^{(\mathrm{as})}(t)= & \frac{f_{0}(0)}{g_{0}(0)}+\sum_{m \neq 0} \frac{i}{m \Omega} \\
& \times\left[f_{m}(-i m \Omega)-g_{m}(-i m \Omega) \frac{f_{0}(0)}{g_{0}(0)}\right] e^{-i m \Omega t} .
\end{aligned}
$$

The dynamics described by (15) can be highly coherent as revealed by a numerical analysis of the Fourier coefficient $P_{1}(\Omega, \hat{\boldsymbol{\epsilon}})$ as a function of the driving frequency: When the external frequency matches fractional values of the intrinsic bias, resonances are found (not shown). At higher frequencies, the spectral amplitude $\eta_{m}$ is completely suppressed, when $\hat{\epsilon} / \Omega$ is a zero of the Bessel function either of order zero $J_{0}(\hat{\epsilon} / \Omega)$ or of $J_{m}(\hat{\epsilon} / \Omega)$. QSR phenomena at high frequency are presently the objects of more detailed investigations.

In summary, we studied novel QSR phenomena in the deep quantum regime. We found that quantum noise can substantially enhance or suppress the nonlinear response. In particular, the occurrence of NIS allows a distortionfree amplification of signals in quantum systems. The possibility of QSR in the presence of coherent tunneling carries a great potential for applications, as they emerge in the task of controlling persistent quantum coherences in complex systems by use of tailored laser pulse sequences [17]. These novel QSR phenomena may be detected by measuring the ac conductance in mesoscopic metals [8,18], in ac-driven atomic force microscopy [19], investigating ac-driven hydrogen tunneling in metals [20], or in driven macroscopic quantum systems [15]. Hence, quantum noise does not represent a nuisance, but rather can be a useful tool when interplaying with external periodic perturbations.

We gratefully acknowledge the support of this work by the Deutsche Forschungsgemeinshaft (HA1517/14-1). We thank R. Bartussek for useful discussions.

[1] R. Benzi, A. Sutera, and A. Vulpiani, J. Phys. A 14, L453 (1981).

[2] See, for example, the Proceedings of the Nato Workshop on SR in Physics and Biology, edited by F. Moss et al. [J. Stat. Phys. 70, 1 (1993)].
[3] For reviews, see F. Moss, D. Pierson, and D. O'Gorman, Int. J. Bifurcation Chaos 4, 1383 (1994); P. Jung, Phys. Rep. 234, 175 (1993).

[4] P. Hänggi, H. Grabert, G. L. Ingold, and U. Weiss, Phys. Rev. Lett. 55, 761 (1985).

[5] Because this quantum correction merely multiplies the classical rate, one finds that the classical SR can be considerably enhanced if the hopping events become assisted by tunneling transitions. M. Grifoni and P. Hänggi (to be published).

[6] M. Grifoni, M. Sassetti, J. Stockburger, and U. Weiss, Phys. Rev. E 48, 3497 (1993).

[7] B. McNamara and K. Wiesenfeld, Phys. Rev. A 39, 4854 (1989).

[8] R. Löfstedt and S. N. Coppersmith, Phys. Rev. Lett. 72, 1947 (1994); Phys. Rev. E 49, 4821 (1994).

[9] R. Bartussek, P. Jung, and P. Hänggi, Phys. Rev. E 49, 3930 (1994).

[10] M. Grifoni, M. Sassetti, P. Hänggi, and U. Weiss, Phys. Rev. E 52, 3596 (1995).

[11] A. J. Leggett, S. Chakravarty, A. T. Dorsey, M.P. A. Fisher, A. Garg, and W. Zwerger, Rev. Mod. Phys. 59, 1 (1987).

[12] U. Weiss, Quantum Dissipative Systems, Series in Modern Condensed Matter Physics Vol. 2 (World Scientific, Singapore, 1993).

[13] The averaged power spectral density $\bar{S}(\omega)$ is defined as the Fourier transform of the averaged correlation function $\bar{C}(\tau)=\frac{\Omega}{2 \pi} \int_{0}^{2 \pi / \Omega} d t\left\langle\sigma_{z}(t+\tau) \sigma_{z}(t)\right\rangle$. Due to the explicit time dependence of the perturbation, the correlation function $\left\langle\sigma_{z}(t+\tau) \sigma_{z}(t)\right\rangle$ depends separately on the time arguments $t$ and $\tau$. The time-averaged function $\bar{C}(\tau)$ is again only a function of the time difference $\tau$.

[14] J. Stockburger, M. Grifoni, and M. Sassetti, Phys. Rev. B 51, 2835 (1995).

[15] R. Rouse, S. Han, and J.E. Lukens, Appl. Phys. Lett. 66, 108 (1995).

[16] Since $\left|J_{m}(z)\right| \leq 1$ for all $m$ and $z$, the coefficients (10) obey the condition $\left|g_{m}\right|<v\left(\epsilon_{0}\right)$ for all $m$. Further, because a fast oscillating external field results in reducing the bath-induced correlations, whenever the NIBA is applicable in the absence of the time-periodic field it is justified even better in the presence of high-frequency driving, i.e., for strong coupling $\alpha>1$ or, for weak coupling, when $k T>\hbar \Delta$ or $\epsilon_{0}>\Delta$.

[17] D. J. Tannor, Nature (London) 369, 445 (1994); P. Brumer and M. Shapiro, Annu. Rev. Phys. Chem. 43, 257 (1992).

[18] B. Golding, N. M. Zimmerman, and S. N. Coppersmith, Phys. Rev. Lett. 68, 998 (1992); K. Chun and N. O. Birge, Phys. Rev. B 48, 11500 (1993).

[19] D. M. Eigler and E. K. Schweitzer, Nature (London) 344, 524 (1990). Here, the quantum dissipation for Xe atoms tunneling between an atomic-force-microscope tip and a $\mathrm{Ni}(100)$ surface is Ohmic-like, and quantum coherent tunneling has indeed been observed experimentally [E. K. Schweitzer (private communication)].

[20] Quantum coherent oscillations with Ohmic coupling have been observed for hydrogen tunneling in niobium by H. Wipf et al., Europhys. Lett. 4, 1379 (1987). 